\title{
Consistent Involvement of Chromosome 13 in Angiolipoma
}

\author{
IOANNIS PANAGOPOULOS ${ }^{1}$, LUDMILA GORUNOVA ${ }^{1}$, KRISTIN ANDERSEN $^{1}$, \\ INGVILD LOBMAIER ${ }^{2}$, BODIL BJERKEHAGEN ${ }^{2}$ and SVERRE HEIM ${ }^{1,3}$ \\ ${ }^{1}$ Section for Cancer Cytogenetics, Institute for Cancer Genetics and Informatics, \\ The Norwegian Radium Hospital, Oslo University Hospital, Oslo, Norway; \\ ${ }^{2}$ Department of Pathology, The Norwegian Radium Hospital, Oslo University Hospital, Oslo, Norway; \\ ${ }^{3}$ Institute of Clinical Medicine, Faculty of Medicine, University of Oslo, Oslo, Norway
}

\begin{abstract}
Background/Aim: Angiolipoma is a rare benign soft tissue tumor composed of mature adipocytes and blood vessels. Genetic information on angiolipomas is scarce. With the single exception of one tumor which carried a $t(X ; 2)(p 22 ; p 12)$, all angiolipomas hitherto investigated cytogenetically had normal karyotypes. Materials and Methods: G-banding chromosome analysis was performed on three short-term cultured angiolipomas. Fluorescence in situ hybridization (FISH) analysis using a commercially available RB1 deletion probe was also done. Results: All three angiolipomas had abnormal karyotypes with loss or structural rearrangement of chromosome 13. The first tumor had the karyotype 46,XY,-6,del(13)(q14),+mar[cp5], the second had 44 45,XY,t(1;10;15)(p21 22;q24;q24),-13[cp5], and the third karyotype was 43,XX,t(13;22;17) (q12;q13; q22 23)[14]. FISH analysis showed heterozygous and homozygous deletion of the RB1 probe in case 2 and 3, respectively. FISH analysis failed in case 1. Conclusion: Chromosome 13 was consistently involved in all three angiolipomas.
\end{abstract}

Angiolipoma is a rare benign soft tissue tumor composed of mature adipocytes and blood vessels (1). Though first described by Bowen in 1912 (2), it was established as a distinct entity in 1960 by Howard and Helwig (3). Angiolipomas typically arise in the subcutaneous tissue of the extremities and trunk of young adults (1) and present as

This article is freely accessible online.

Correspondence to: Ioannis Panagopoulos, Section for Cancer Cytogenetics, Institute for Cancer Genetics and Informatics, The Norwegian Radium Hospital, Oslo University Hospital, Montebello, PO Box 4954 Nydalen, NO-0424 Oslo 0310, Norway. Tel: +47 22782363, e-mail: ioannis.panagopoulos@rr-research.no

Key Words: Angiolipoma, cytogenetics, chromosome 13, deletion, $R B 1$ gene. tender to painful single or multiple subcutaneous nodules. On gross pathology, angiolipoma is a circumscribed, yellowred nodular mass with high fat content and blood vessel proliferation. Microscopically, the tumor consists of mature adipocytes and clusters of small vessels, predominantly in the periphery, and often with fibrin microthrombi in the vessel lumen. The relative proportion of adipose tissue and vessels varies from case to case but mostly the adipose tissue predominates (1).

Genetic information on angiolipomas is scarce (1). With the single exception of one tumor which had the abnormal karyotype 46,Y,t(X;2)(p22;p12), all angiolipomas investigated so far had normal karyotypes (4-6).

We present, here, cytogenetic data on three angiolipomas, all of which turned out to have abnormal karyotypes with involvement of chromosome 13 .

\section{Materials and Methods}

Ethics statement. The study was approved by the Regional Committee for Medical and Health Research Ethics, South-East Norway (REK Sør-Øst; http://helseforskning.etikkom.no) and written informed consent was obtained from the patients. The ethics committee's approval included a review of the consent procedure. All patient information has been anonymized.

Patients and tumors. Angiolipomas from three patients were studied. Data concerning patients' gender and age as well as tumor location and size are shown in Table I. Figure 1 shows a microscopic picture after hematoxylin and eosin staining of the angiolipoma of case 1. The pathologic findings were similar in cases 2 and 3 .

G-banding, karyotyping, and fluorescence in situ hybridization (FISH). Fresh tissue from a representative area of the tumors was received and analyzed cytogenetically as part of our diagnostic routine. The samples were disaggregated mechanically and enzymatically with collagenase II (Worthington, Freehold, NJ, USA). The resulting cells were cultured and harvested using standard techniques (7). Chromosome preparations were G-banded with Wright's stain (Sigma Aldrich; St Louis, MO, USA) and examined. 
Table I. Clinicopathological data and karyotypes of the three angiolipomas.

\begin{tabular}{lcccc}
\hline Cases & Gender/Age & Location of the tumor & Size $(\mathrm{cm})$ & Karyotype \\
\hline 1 & $\mathrm{M} / 57$ & Right shoulder & $15 \times 11 \times 4$ & $46, \mathrm{XY},-6, \operatorname{del}(13)(\mathrm{q} 14),+\mathrm{mar}[\mathrm{cp} 5] / 46, \mathrm{XY}[16]$ \\
2 & $\mathrm{M} / 54$ & Right foot & $8 \times 4.5 \times 2$ & $44 \sim 45, \mathrm{XY}, \mathrm{t}(1 ; 10 ; 15)(\mathrm{p} 21 \sim 22 ; \mathrm{q} 24 ; \mathrm{q} 24),-13[\mathrm{cp} 5] / 46, \mathrm{XY}[10]$ \\
3 & $\mathrm{~F} / 28$ & Left shoulder/back & $8.5 \times 4.5$ & $43, \mathrm{XX}, \mathrm{t}(13 ; 22 ; 17)(\mathrm{q} 12 ; \mathrm{q} 13 ; \mathrm{q} 22 \sim 22)[14]$ \\
\hline
\end{tabular}

Metaphases were analyzed and karyograms prepared using the CytoVision computer assisted karyotyping system (Leica Biosystems, Newcastle, UK). The karyotypes were described according to the International System for Human Cytogenetic Nomenclature (8).

Interphase and metaphase FISH analyses were performed in order to detect deletion of the $R B 1$ locus in $13 \mathrm{q} 14.2$. The $R B 1$ deletion probe, purchased from Cytocell (http://www.cytocell.com/), consisted of a $318 \mathrm{~kb}$ red probe spanning the RB1 gene locus and a 13qter probe in green acting as a control for chromosome 13. Fluorescent signals were captured and analyzed using the CytoVision system from Leica Biosystems (http://www.leicabiosystems.com/clinical-microscopysurgery-radiology/cytogenetics/).

\section{Results}

All 3 angiolipomas had abnormal karyotypes with loss or structural rearrangement of chromosome 13 (Table I). In case 1 , there was a del(13)(q14) together with loss of chromosome 6 and a marker chromosome (Figure 2). In case 2, an entire chromosome 13 was lost accompanied by a three-way

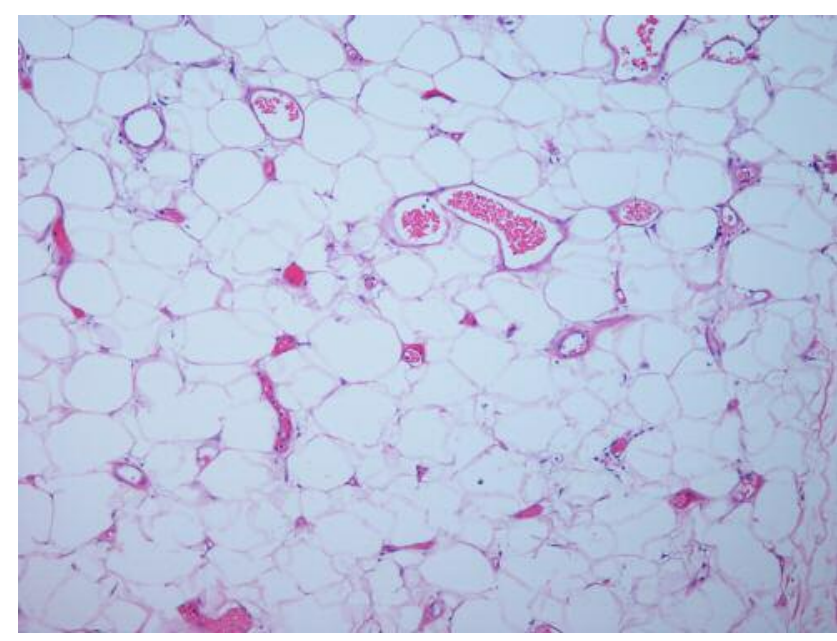

Figure 1. H\&E-stained histological image of an angiolipoma ( $x 10$; case 1$)$ with characteristic small vessels predominately in the periphery, focally with small thrombi.

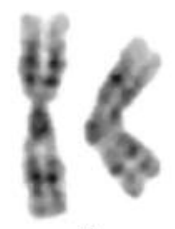

1

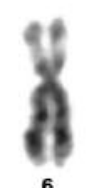

6

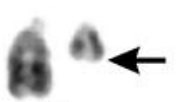

13

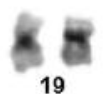

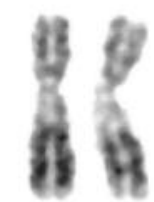

2

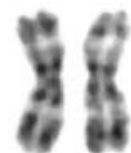

3

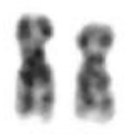

9

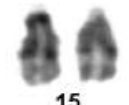

15
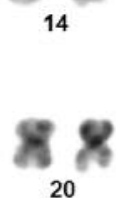

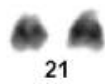

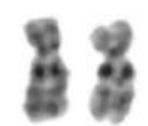

10

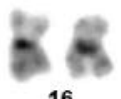

16

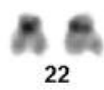

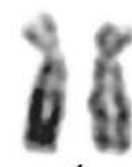

4

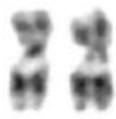

11

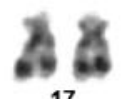

17

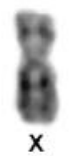

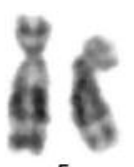

5

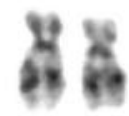

12

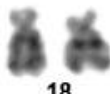

18

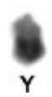

Figure 2. Karyotype of angiolipoma (case 1) showing del(13)(q14), loss of chromosome 6, and a marker chromosome (mar). Arrow indicates breakpoint. 

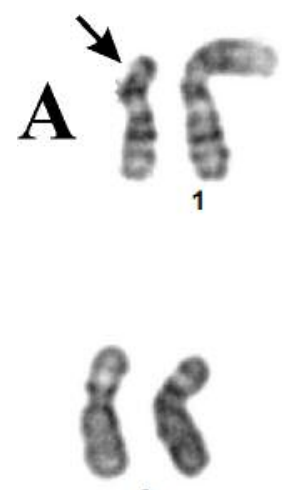

6

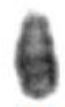

13

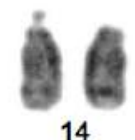

14

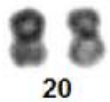

19

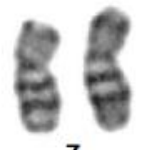

7

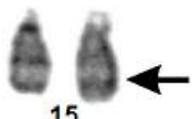

15

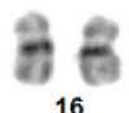

16

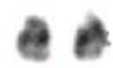

21

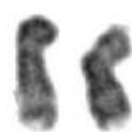

9

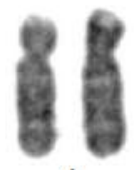

4

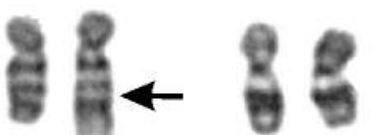

11

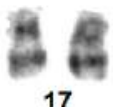

17

18

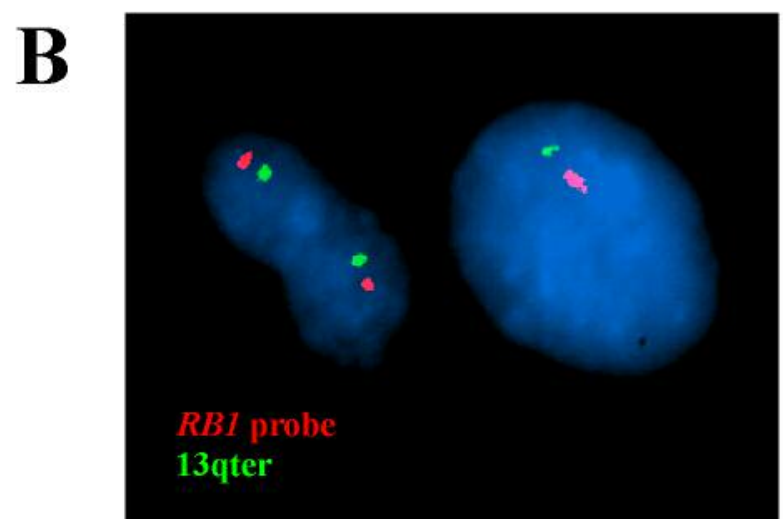

Figure 3. Cytogenetic analysis of the angiolipoma of case 2. A) Karyotype showing loss of chromosome 13 and the three-way translocation $t(1 ; 10 ; 15)(p 21 \sim 22 ; q 24 ; q 24)$. Arrows indicate breakpoints. B) Interphase FISH analysis demonstrating heterozygous deletion of RB1.

translocation $\mathrm{t}(1 ; 10 ; 15)(\mathrm{p} 21 \sim 22 ; \mathrm{q} 24 ; \mathrm{q} 24)$ (Figure 3A). Case 3 had a three-way translocation, $\mathrm{t}(13 ; 22 ; 17)(\mathrm{q} 12 ; \mathrm{q} 13 ; \mathrm{q} 22 \sim 23)$, as the sole cytogenetic abnormality (Figure 4 ).

In case 2, FISH analysis showed heterozygous deletion of the $R B 1$ probe in 50 out of 100 investigated interphase nuclei
(Figure 3B). In case 3, FISH analysis showed homozygous deletion of $R B 1$ in 98 out of 100 interphase nuclei (Figure 4B). The same result, i.e., homozygous deletion of the $R B I$ probe, was also seen in metaphase spreads (Figure 4B). FISH analysis failed for case 1 . 


\section{Discussion}

The present study shows consistent involvement of chromosome 13 in all three angiolipomas studied. The tumors of cases 1 and 3 arose in the shoulder and had structural aberrations of chromosome 13 affecting the q12-q14 bands. In the third tumor (case 2), which was located in the foot, one entire chromosome 13 was lost. In the tumors of cases 1 and 2, the loss of material from chromosome 13 was accompanied by other chromosome aberrations whereas the tumor of case 3 had a three-way translocation as the sole cytogenetic abnormality.

Our results are at odds with previously published data. Mandahl et al. (5) reported that in one patient with five subcutaneous angiolipomas, four of 19 metaphases of one tumor showed $\mathrm{t}(\mathrm{X} ; 2)(\mathrm{p} 22 ; \mathrm{p} 12)$ as the sole anomaly whereas the four remaining tumors were karyotypically normal. Sciot et al. (6) reported that all 20 subcutaneous angiolipomas occurring in 10 patients showed a normal karyotype based on analysis of a minimum of 20 metaphases. The authors took the normal karyotype to be indicative of a non-neoplastic nature of the lesions, thus supporting the clinicopathologic impression of angiolipomas as being reactive or hamartomatous. The findings of the present study, that acquired clonal chromosome abnormalities exist in cells cultured from three tumors from three different patients, confirm that angiolipomas are neoplastic in nature. Furthermore, the consistent involvement of chromosome 13, with structural aberrations affecting bands 13q12-14 in cases 1 and 3 and deletion of the RBI locus found by FISH, suggest a (cyto)genetic similarity between angiolipoma and spindle cell lipoma, cellular angiofibroma, and extramammary myofibroblastoma $(4,9-13)$. Most of the 28 karyotypically abnormal spindle cell lipomas reported in the cytogenetic literature had loss of material from 13q together with other chromosome abnormalities (http://cgap.nci.nih.gov/ Chromosomes/Mitelman, Database last updated on August 11, 2016), with bands $13 q 12 \sim 21$ or $13 q 12-22$ being the most commonly lost $(4,9,10)$. Similarly, G-banding analysis has shown loss of $13 \mathrm{q}$ material to be typical of cellular angiofibromas (14).

The deletions of the RBI locus found by FISH in cases 2 (heterozygous deletion) and 3 (homozygous deletion) add evidence pointing in the same direction: Mono- or bi-allelic deletions of $R B 1$ were found also in spindle cell lipomas, cellular angiofibromas, and extramammary myofibroblastomas $(4,9,11-15)$. At the protein level, immunohistochemical staining for RB1 showed that nuclear RB1 expression was deficient in all examined spindle cell lipomas, pleomorphic lipomas, and cellular angiofibromas, and in nearly $90 \%$ (17 of 19) of mammary-type myofibroblastomas (16). The protein encoded by the $R B 1$ gene regulates transcription and is a negative regulator of cell proliferation (17). At present, its exact role, if any, in the development of the above-mentioned tumors, angiolipomas included, remains unknown.
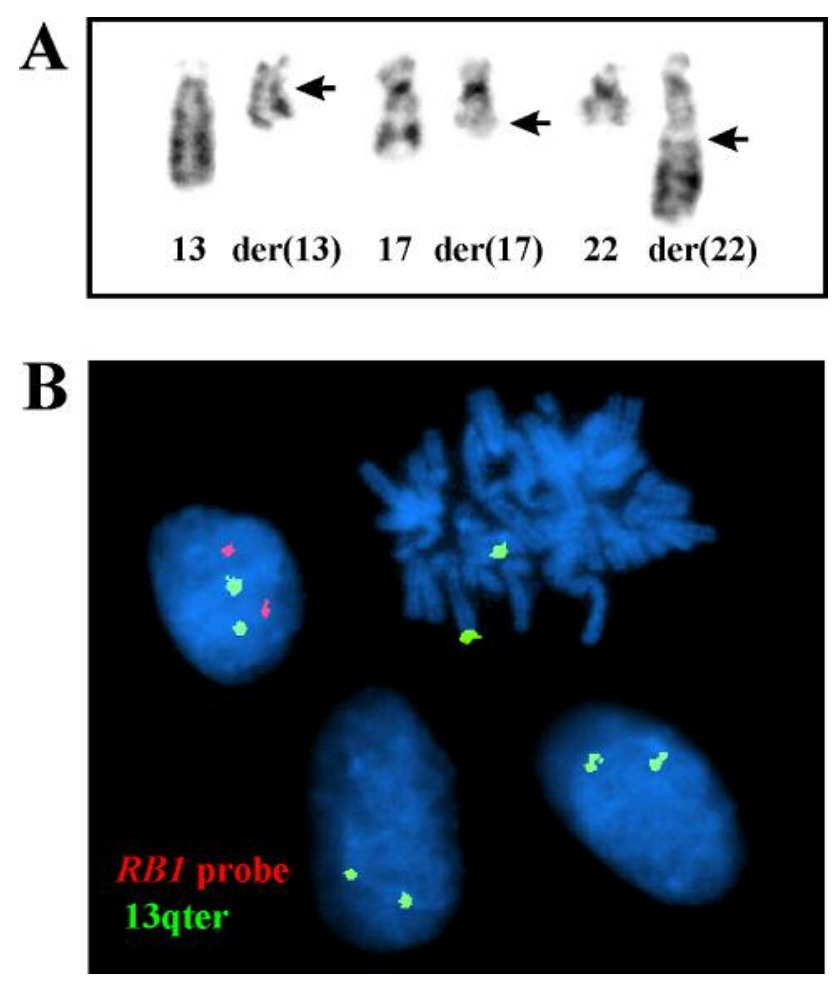

Figure 4. Cytogenetic analysis of the angiolipoma of case 3. A) Partial karyotype showing the $\operatorname{der}(13), \operatorname{der}(17)$, and $\operatorname{der}(22)$ of the three-way translocation $t(13 ; 22 ; 17)(q 12 ; q 13 ; q 22 \sim 23)$ together with their corresponding normal chromosome homologs. Arrows indicate breakpoints. B) FISH analysis showing a normal nucleus, two nuclei with biallelic deletion of RB1, and a metaphase spread with biallelic deletion of RB1.

In conclusion, chromosome 13 was consistently involved in all three angiolipomas.

\section{Conflicts of Interest}

The Authors declare that they have no competing interests.

\section{References}

1 Sciot $\mathrm{R}$ and Mandahl $\mathrm{N}$ : Angiolipoma. In: World Health Organization Classification of Tumours. Pathology and Genetics of Tumours of Soft Tissue and Bone. Fletcher CDM, Bridge JA, Hogendoorn PCW, Mertens F (eds.). Lyon, IARC Press, pp. 26, 2013.

2 Bowen JT: Multiple subcutaneous hemangiomas, together with multiple lipomas, occurring in enormous numbers in an otherwise healthy, muscular subject. Am J Med Sci 144: 189192, 1912.

3 Howard WR and Helwig EB: Angiolipoma. Arch Dermatol 82: 924-931, 1960.

4 Bartuma H, Nord KH, Macchia G, Isaksson M, Nilsson J, Domanski HA, Mandahl N and Mertens F: Gene expression and 
single nucleotide polymorphism array analyses of spindle cell lipomas and conventional lipomas with 13q14 deletion. Genes Chrom Cancer 50: 619-632, 2011.

5 Mandahl N, Höglund M, Mertens F, Rydholm A, Willen H, Brosjö O and Mitelman F: Cytogenetic aberrations in 188 benign and borderline adipose tissue tumors. Genes Chrom Cancer 9: 207-215, 1994.

6 Sciot R, Akerman M, Dal Cin P, De Wever I, Fletcher CD, Mandahl N, Mertens F, Mitelman F, Rosai J, Rydholm A, Tallini $\mathrm{G}$, Van den Berghe $\mathrm{H}$, Vanni R and Willen $\mathrm{H}$ : Cytogenetic analysis of subcutaneous angiolipoma: further evidence supporting its difference from ordinary pure lipomas: a report of the CHAMP Study Group. Am J Surg Pathol 21: 441-444, 1997.

7 Mandahl N: Methods in solid tumour cytogenetics. In: Human cytogenetics: malignancy and acquired abnormalities. Rooney DE (eds.). New York, Oxford University Press, pp. 165-203, 2001 .

8 Schaffer LG, McGowan-Jordan J and Schmid M: ISCN 2013: An International System for Human Cytogenetic Nomenclature Basel, Karger, 2013.

9 Dahlén A, Debiec-Rychter M, Pedeutour F, Domanski HA, Höglund M, Bauer HC, Rydholm A, Sciot R, Mandahl N and Mertens F: Clustering of deletions on chromosome 13 in benign and low-malignant lipomatous tumors. Int J Cancer 103: 616623, 2003.

10 Dal Cin P, Sciot R, Polito P, Stas M, de Wever I, Cornelis A and Van den Berghe $\mathrm{H}$ : Lesions of 13q may occur independently of deletion of $16 \mathrm{q}$ in spindle cell/pleomorphic lipomas. Histopathology 31: 222-225, 1997.

11 Hameed M, Clarke K, Amer HZ, Mahmet K and Aisner S: Cellular angiofibroma is genetically similar to spindle cell lipoma: a case report. Cancer Genet Cytogenet 177: 131-134, 2007
12 Maggiani F, Debiec-Rychter M, Vanbockrijck M and Sciot R: Cellular angiofibroma: another mesenchymal tumour with 13q14 involvement, suggesting a link with spindle cell lipoma and (extra)-mammary myofibroblastoma. Histopathology 51: 410412, 2007.

13 Maggiani F, Debiec-Rychter M, Verbeeck G and Sciot R: Extramammary myofibroblastoma is genetically related to spindle cell lipoma. Virchows Arch 449: 244-247, 2006.

14 Panagopoulos I, Gorunova L, Bjerkehagen B, Andersen K, Lund-Iversen $\mathrm{M}$ and Heim S: Loss of chromosome 13 material in cellular angiofibromas indicates pathogenetic similarity with spindle cell lipomas. Diagn Pathol 12: 17, 2017.

15 Flucke U, van Krieken JH and Mentzel T: Cellular angiofibroma: analysis of 25 cases emphasizing its relationship to spindle cell lipoma and mammary-type myofibroblastoma. Mod Pathol 24: 82-89, 2011.

16 Chen BJ, Marino-Enriquez A, Fletcher CD and Hornick JL: Loss of retinoblastoma protein expression in spindle cell/pleomorphic lipomas and cytogenetically related tumors: an immunohistochemical study with diagnostic implications. Am J Surg Pathol 36: 1119-1128, 2012.

17 Dyson NJ: RB1: a prototype tumor suppressor and an enigma. Genes Dev 30: 1492-1502, 2016.
Received October 24, 2017

Revised November 10, 2017

Accepted November 13, 2017 